\title{
Tocilizumab Effects on Coagulation Factor XIII in Patients with Rheumatoid Arthritis
}

\author{
Roberta Gualtierotti (D) - Francesca Ingegnoli • Massimo Boscolo • \\ Samantha Griffini · Elena Grovetti · Massimo Cugno
}

Received: September 4, 2019 / Published online: October 25, 2019

(c) The Author(s) 2019

\begin{abstract}
Introduction: Rheumatoid arthritis (RA) is a chronic systemic auto-immune disease associated with a prothrombotic state. Tocilizumab, an interleukin-6 receptor inhibitor, is highly effective in controlling disease activity and thrombotic risk. Factor XIII (FXIII), involved in thrombotic complications, has been reported to be reduced in RA patients during maintenance treatment with tocilizumab, but no data are available before and after the drug administration. Thus, we investigated the effects of tocilizumab on FXIII, thrombin generation and
\end{abstract}

Enhanced Digital Features To view enhanced digital features for this article go to: https://doi.org/10.6084/ m9.figshare.9944459.

R. Gualtierotti $(\bowtie)$

Dipartimento di Biotecnologie Mediche e Medicina

Traslazionale, Università degli Studi di Milano,

Milan, Italy

e-mail: roberta.gualtierotti@unimi.it

F. Ingegnoli

UOC Reumatologia Clinica, ASST Pini-CTO, Dipartimento di Scienze Cliniche e di Comunità,

Università degli Studi di Milano, Milan, Italy

M. Boscolo · S. Griffini · E. Grovetti · M. Cugno Medicina Interna, Dipartimento di Fisiopatologia Medico-Chirurgica e dei Trapianti, Ospedale Maggiore Policlinico, IRCCS Fondazione $\mathrm{Ca}^{\prime}$ Granda Milano, Università degli Studi di Milano, Milan, Italy inflammation in patients with RA naïve for the drug.

Methods: We studied 15 consecutive adult patients with RA at baseline and 4 weeks after the onset of parenteral administration of tocilizumab, measuring disease activity and plasma levels of C-reactive protein (CRP), FXIII, and prothrombin fragments $\mathrm{F} 1+2$ by immunoenzymatic methods. Fifteen healthy subjects, sexand age-matched with patients, served as normal controls for laboratory measurements.

Results: At baseline, patients with established RA had a median DAS28 of 4.8 (3.2-8.3) and, compared to healthy controls, had higher plasma levels of CRP $(p<0.0001)$, FXIII $(p=0.017)$ and $\mathrm{F} 1+2(p<0.0001)$. Four weeks after starting treatment with tocilizumab, based on the EULAR response criteria, eight patients were classifiable as responders and seven as nonresponders. In responders, we observed a statistically significant reduction not only of the values of DAS28 and CRP ( $p=0.012$ for both), ut also of plasma levels of FXIII $(p=0.05)$ and $\mathrm{F} 1+2(p=0.025)$. In non-responders, all the studied parameters were unchanged.

Conclusion: The decrease of FXIII and F1+2 levels after tocilizumab treatment observed only in those patients who responded to the drug indicates that the effect of tocilizumab on the prothrombotic state is linked to the control of inflammation and disease activity and not to a direct effect of the drug, thus contributing to the reduction of the cardiovascular risk. 
Keywords: Coagulation; $\quad$ Factor XIII; Inflammation; Rheumatoid arthritis; Rheumatology; Tocilizumab

\section{Key Summary Points}

Why carry out this study?

Rheumatoid arthritis (RA) is a chronic systemic auto-immune disease associated with a prothrombotic state whose pathogenesis is multifactorial and not completely elucidated.

Factor XIII (FXIII), which is involved not only in blood coagulation but also in inflammation and in bone/cartilage metabolism, has been reported to be reduced in patients in maintenance treatment with tocilizumab.

We studied patients with RA naïve for tocilizumab before and after drug administration and measured FXIII levels and the degree of inflammation/disease activity, and the prothrombin fragment $\mathrm{F} 1+2$, a known marker of prothrombotic state.

\section{What was learned from the study?}

We observed a decrease of FXIII and F1+2 levels after tocilizumab treatment only in patients who responded to the drug, thus indicating that the reduction of the prothrombotic state exerted by tocilizumab is linked to the regulation of inflammation and disease activity and not to a direct effect of the drug.

The decrease of FXIII by tocilizumab may contribute to the reduction of the cardiovascular risk that is one of the major causes of morbidity and mortality in RA patients.

\section{INTRODUCTION}

Rheumatoid arthritis (RA) is a chronic systemic auto-immune disease characterized not only by joint involvement but also by systemic inflammation, which has been associated with a prothrombotic state responsible for an increased cardiovascular mortality [1-3].

The control of chronic inflammation by treatment with disease-modifying anti-rheumatic drugs (DMARDs) has been associated with a reduced cardiovascular risk [4]. Over the last 20 years, the therapeutic management of RA has been revolutionized by the introduction of biological DMARDs, which seem more effective than conventional synthetic DMARDs, not only in the control of disease but also in reducing cardiovascular risk $[3,5,6]$. In particular, these effects seem to be major with tocilizumab-a human monoclonal antibody directed against interleukin-6 (IL-6) receptor-than with inhibitors of tumor necrosis factor (TNF) [7, 8]. Furthermore, tocilizumab has been reported to reduce plasma levels of factor XIII (FXIII) [9-11]. FXIII is a tetrameric zymogen consisting of two potentially active subunits A (FXIIIA) and two inhibitory/carrier subunits B (FXIIIB). It is activated by the concerted action of thrombin and $\mathrm{Ca} 2+$ in the terminal phase of the clotting cascade. After cleavage by thrombin in the presence of $\mathrm{Ca} 2+$, FXIIIB dissociates, and the FXIIIA dimer becomes active. Activated FXIII is a transglutaminase that is able to cross-link the $\gamma$ - and $\alpha$-chains of fibrin, thus leading to the stabilization of fibrin clots, which become more resistant to fibrinolysis [12]. The important involvement of FXIII in the coagulation cascade is supported by the evidence that FXIII deficiency is associated with a bleeding syndrome, whereas an excess of FXIII has been associated with thrombosis [13, 14]. Finally, evidence in experimental models of RA supports the involvement of FXIII in cartilage and bone destruction $[15,16]$, showing that the reduction of FXIII activity leads to improvement of cartilage and bone lesions [15].

To the best of our knowledge, no evidence of the effect of tocilizumab on FXIII levels is currently available in patients with active RA naïve to this treatment. Therefore, the aim of our study was to investigate the effect of tocilizumab on FXIII in such patients and to evaluate its correlation with clinical response, prothrombotic parameters and inflammation. 


\section{METHODS}

\section{Patient Population}

We studied 15 consecutive adult patients with RA (13 women and 2 men), median age 57 years (min 30-max 72 years) attending the out-patient clinic of Gaetano Pini Institute in Milan, Italy, from July 2017 to January 2019, and fulfilling 2010 American College of Rheumatology (ACR) criteria for RA [17], naïve for tocilizumab. Demographic and clinical characteristics of the study population are reported in Table 1. Patients treated with oral anticoagulants and patients with other diseases of coagulation were excluded. The sample size was calculated in order to obtain a statistical power of $80 \%$, with an alpha error of $5 \%$, based on the study by Mokuda et al., which showed a $40 \%$ decrease in FXIII levels in patients treated with tocilizumab compared to normal subjects [10]. Furthermore, the sample size was confirmed based on our previous study, showing a decrease of $\mathrm{F} 1+2$ levels of around $60 \%$ in patients treated with tocilizumab compared with baseline levels [8].

At baseline and 4 weeks after the onset of subcutaneous administration of tocilizumab
(162 mg weekly), disease activity was measured and blood samples were collected for measurement of C-reactive protein (CRP), FXIII, and prothrombin fragments $\mathrm{F} 1+2$. For the same parameters, 15 healthy subjects sex- and agematched with patients served as normal controls. The control group consisted of 13 women and 2 males, median age 55 years (min 28-max 73 years); a smoking habit was reported by one subject.

The authors received Ethics Committee approval on 20 July 2017 (Fondazione IRCCS $\mathrm{Ca}^{\prime}$ Granda Ospedale Maggiore Policlinico di Milano, no. 484_2017). The study conformed with the Helsinki Declaration of 1964, as revised in 2013, concerning human and animal rights, and Springer's policy concerning informed consent has been followed.

\section{Disease Activity Assessment}

Disease activity was measured based on the evaluation of 28 joints and erythrocyte sedimentation rate (DAS28) [18]. Response to tocilizumab was assessed on the basis of the European League Against Rheumatism (EULAR) response criteria [19], in particular, good

Table 1 Demographic and clinical characteristics of the study population

\begin{tabular}{ll}
\hline Total RA patients $(n)$ & 15 \\
Median age (min-max) & $57(30-72)$ years \\
Median disease duration (min-max) & $10.7(1.5-35.1)$ years \\
Females \% $(n)$ & $86.7 \%(13)$ \\
Smoking habit \% $(n)$ & $13.3 \%(2)$ \\
RF positivity \% $(n)$ & $60 \%(9)$ \\
ACPA positivity \% $(n)$ & $66.7 \%(10)$ \\
Daily equivalent prednisone dose $\geq 5 \mathrm{mg} /$ day $\%(n)$ & $73.3 \%(11)$ \\
Mean prednisone daily dose & $4.5 \mathrm{mg}$ \\
Weekly MTX dose $\geq 10 \mathrm{mg} /$ week $\%(n)$ & $33.3 \%(5)$ \\
Mean MTX weekly dose & $12 \mathrm{mg}$ \\
Median number of previous biologic drugs (min-max) & $2(0-6)$ \\
\hline
\end{tabular}

$R A$ rheumatoid arthritis, $R F$ rheumatoid factor, $A C P A$ anti-citrullinated protein antibodies, $M T X$ methotrexate 
responders were considered patients with DAS28 scores of 3.2 or less with reductions in DAS28 of more than 1.2. Patients with DAS28 scores over 3.2 who have reductions in DAS28 scores of more than 1.2 have moderate responses. Patients with reductions in DAS28 of less than 0.6 are non-responders.

\section{Plasma Measurement}

CRP was measured with a sandwich enzymelinked immunosorbent assay (ELISA; Zymutest CRP; Hyphen BioMed, Neuville-sur-Oise, France). Intra- and inter-assay coefficients of variation $(\mathrm{CV})$ were lower than $11 \%$.

FXIII levels were measured in plasma samples by means of an ELISA (HemosIL Factor XIII Antigen; Instrumentation Laboratory, Bedford, MA, USA) is expressed as the \% of normal. The method is highly specific for the potentially active subunit A of FXIII. The intra- and interassay CV is lower than $8.1 \%$.

Prothrombin fragment $\mathrm{F} 1+2$ levels were measured in plasma using an ELISA (Enzygnost F1+2; Siemens Healthcare Diagnostics, Marburg, Germany), with intra- and inter-assay CVs of $5 \%$ and $8 \%$, respectively.

\section{Statistical Analysis}

Since the data were positively skewed and the test of Kolmogorov-Smirnov excluded a normal distribution, we have reported the results as median (minimum-maximum) values. The Mann-Whitney $U$ test was used to compare different groups (healthy vs. RA) and Wilcoxon test for paired samples to compare the baseline with 4 -week values in RA patients. Correlations were calculated by means of Spearman's rho. Significance level was set at $p<0.05$. Data were analyzed using the SPSS PC statistical package v.25.0 (IBM, Armonk, NY, USA).

\section{RESULTS}

At baseline, patients with RA compared with healthy controls had significantly higher median plasma levels of CRP: $8.9 \mu \mathrm{g} / \mathrm{mL}$
$(0.1-17.9 \mu \mathrm{g} / \mathrm{mL})$ versus $0.9 \mu \mathrm{g} / \mathrm{mL}(0.2-4.1 \mu \mathrm{g} /$ $\mathrm{mL}, p<0.0001)$; FXIII: $146 \%(81-197)$ versus $105 \%(74-139, p=0.017) ; \mathrm{F} 1+2: 342 \mathrm{pmol} / \mathrm{mL}$ (211-1483 pmol $/ \mathrm{mL}) \quad$ vversus $180 \mathrm{pmol} / \mathrm{mL}$ $(116-283 \mathrm{pmol} / \mathrm{mL}, p<0.0001)$. DAS28 median levels were 4.8 (3.2-8.3).

After 4 weeks from the onset of tocilizumab treatment, 8 patients were responders, according to the EULAR response criteria [19], and 7 patients were non-responders. At baseline, there were no differences between responders and non-responders in terms of DAS28, CRP, FXIII and $\mathrm{F} 1+2$. None of the patients experienced bleeding complications. No changes in medications were reported during the study period.

As expected, in responders, we observed a significant decrease of median DAS28 and of CRP plasma levels ( $p=0.012$ for both; Fig. 1 ; Table 2). Furthermore, we observed a reduction of median plasma levels of FXIII $(p=0.05$; Fig. 2; Table 2) and of F1+2 ( $p=0.025$; Fig. 3; Table 2 ). In non-responders, all these parameters were not significantly different after 4 weeks compared to baseline (Figs. 1, 2, 3; Table 2).

Then, we sought to find correlations between the different clinical and laboratory parameters and found significant correlations between DAS28 and CRP levels $(r=0.63, p=0.01)$ as expected, between CRP and F1+2 levels $(r=0.57, p=0.001)$ and between FXIII and F1+2 levels $(r=0.46, p=0.01)$, supporting the strict relationship between the activity of the disease and the prothrombotic parameters.

\section{DISCUSSION}

To the best of our knowledge, this is the first study showing that tocilizumab reduces the levels of the prothrombotic biomarker $\mathrm{F} 1+2$ and FXIII subunit $\mathrm{A}$, which in RA patients with active disease are higher than in healthy controls. This effect is already observed after 4 weeks in patients who clinically respond to the drug. Thus, our data indicate that IL-6 signaling blockade by tocilizumab may reduce the prothrombotic state that is well known in RA, as shown by a number of studies from several authors and from our group $[3,5,6,8,20]$. 


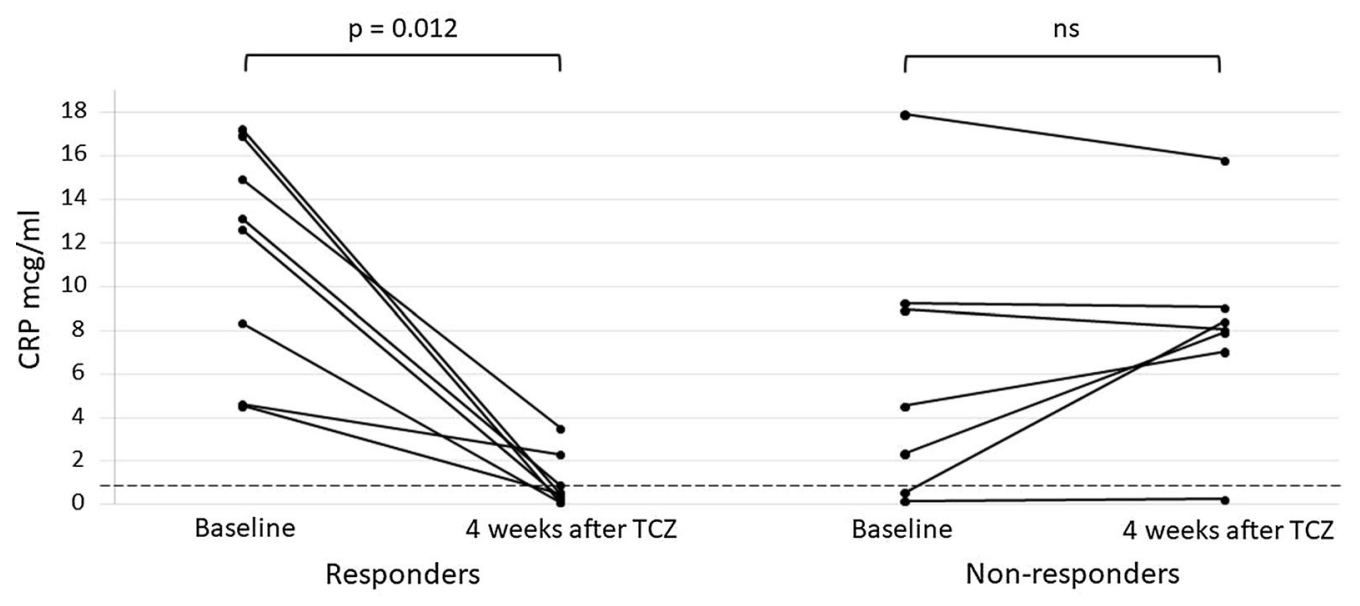

Fig. 1 Plasma levels of C-reactive protein $(C R P)$ in rheumatoid arthritis patients treated with tocilizumab $(T C Z)$ at baseline and after 4 weeks, divided based on the EULAR response criteria. Responders showed a

significant reduction of CRP plasma levels, whereas in non-responders the levels were not significantly (ns) different. The dashed line represents the median value of normal subjects

Table 2 Disease activity $(D A S 28)$ and plasma levels of C-reactive protein (CRP), factor XIII (FXIII) and prothrombin fragment $\mathrm{F} 1+2$ in 15 patients with rheumatoid arthritis treated with tocilizumab at baseline and after 4 weeks, divided based on the EULAR response criteria

\begin{tabular}{lllllll}
\hline & Healthy controls & Responders & & & \multicolumn{2}{l}{ Non-responders } \\
& & Baseline & After 4 weeks & & Baseline & After 4 weeks \\
\hline DAS28 & - & $5.1(3.2-6.3)$ & $1.8(0.9-2.9)^{* * *}$ & & $4.4(3.5-8.3)$ & $4.3(3.4-7.5)$ \\
CRP $\mu \mathrm{g} / \mathrm{ml}$ & $0.9(0.2-4.1)$ & $12.9(4.5-17.2)$ & $0.5(0.1-3.5)^{* * *}$ & & $4.5(0.1-17.9)$ & $8.0(0.2-15.8)$ \\
FXIII \% & $105(74-139)$ & $131(95-166)$ & $112(93-133)^{*}$ & & $148(81-197)$ & $156(90-190)$ \\
F1+2 $\mathrm{pmol} / \mathrm{ml}$ & $180(116-283)$ & $354(317-1483)$ & $330(146-536)^{* *}$ & & $278(211-751)$ & $277(181-396)$ \\
\hline
\end{tabular}

Values are expressed as median with minimum and maximum in parentheses

Statistical significance: ${ }^{* *} p=0.012 ;{ }^{* *} p=0.025 ;{ }^{*} p=0.05$

As far as we know, to date, higher levels of FXIII during the acute phase of RA have never been reported, and we did not find any study in the literature describing FXIII as an acute phase reactant. Indeed, in our active RA patients, the levels of FXIII before the administration of tocilizumab were significantly higher than in normal controls. High FXIII levels have been demonstrated to be associated with cardiovascular diseases, such as myocardial infarction and obliterative atherosclerosis of the lower limb, as well as with diabetes [21-26], whereas lower FXIII levels, such as those observed in patients with some polymorphisms in their FXIII genes, decrease the risk of coronary artery disease $[21,22,27]$. Therefore, the lowering effect of tocilizumab on FXIII levels may be a further mechanism of reduction of the cardiovascular risk. It is well known that the congenital FXIII deficiency is associated with a hemorrhagic syndrome characterized by impaired wound healing, recurrent miscarriages, bleeding of soft tissues, and life-threatening central nervous system bleeding, for which a replacement therapy is currently available [28]. The acquired FXIII deficiency, 


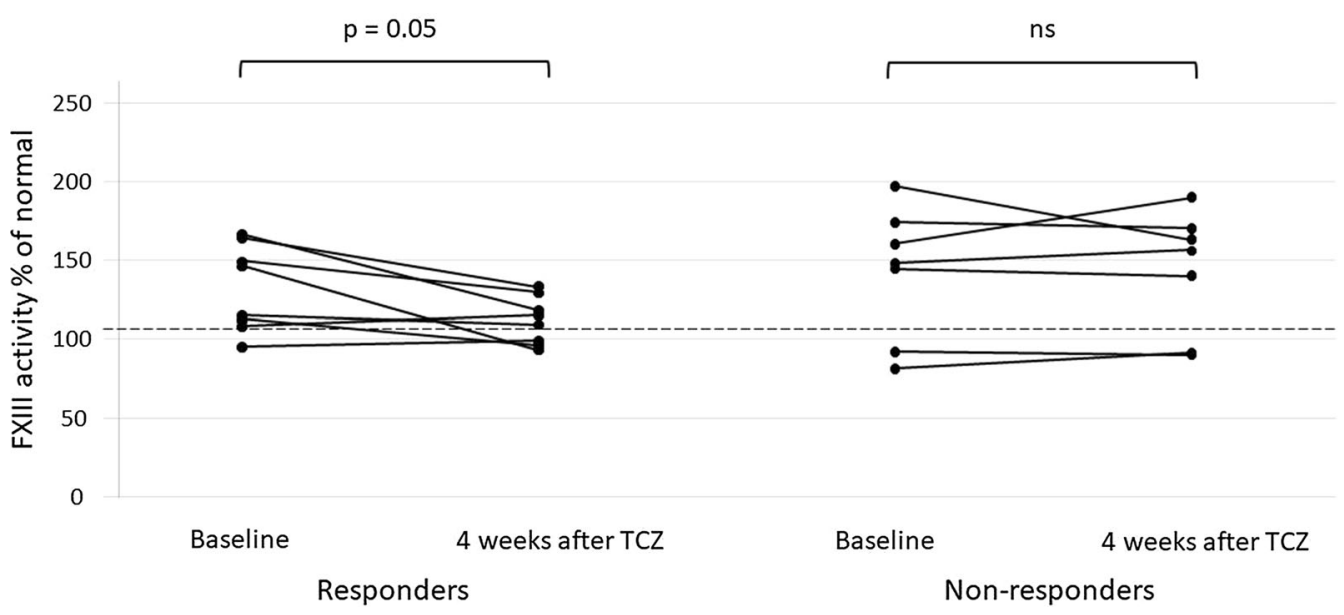

Fig. 2 Plasma levels of factor XIII (FXIII) in rheumatoid arthritis patients treated with tocilizumab $(T C Z)$ at baseline and after 4 weeks, divided based on the EULAR response criteria. Responders showed a significant

reduction of FXIII plasma levels, whereas in non-responders the levels were not significantly (ns) different. The dashed line represents the median value of normal subjects

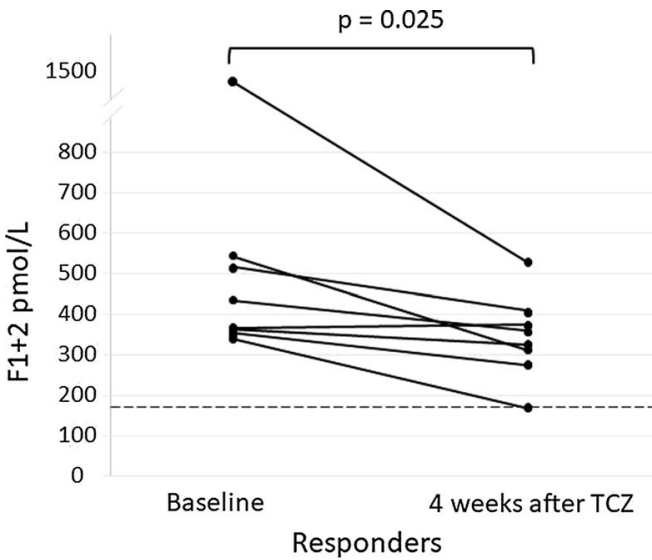

Fig. 3 Plasma levels of prothrombin fragment $F 1+2$ in rheumatoid arthritis patients treated with tocilizumab $(T C Z)$ at baseline and after 4 weeks, divided based on the EULAR response criteria. Responders showed a significant

which is due to the production of auto-antibodies in the course of several conditions, such as auto-immune diseases or malignancy, is an emerging disorder [29]. An acquired FXIII deficiency not due to auto-antibody production has also been reported in a few Japanese patients with RA treated with tocilizumab $[9,11]$, but, to the best of our knowledge, it has never been reported in Caucasian patients.
Besides its role in the final stage of the coagulation cascade, FXIII has been shown to have several other biological functions. In particular, it is involved in cartilage and bone metabolism and inflammation, as demonstrated by experimental studies in which reducing FXIII levels provide protection from inflammation and bone erosions in an induced arthritis model in mice [15]. Intriguingly, in an 
observational study in patients with RA treated with tocilizumab monotherapy, a consistent improvement of existing bone erosions has been demonstrated [30]. The mechanism underlying the reduction of FXIII induced by tocilizumab is presently unknown. However, as the production of FXIII from monocytes of the synovial membrane of RA patients is high [31], one can expect that the reduction of the inflammatory stimulation on the synovial membrane may play a role in decreasing the production and release of FXIII. Indeed, Mokuda et al. also found reduced FXIII levels in patients treated with TNF inhibitors, but this reduction did not reach the magnitude observed with tocilizumab [10].

F1+2 plasma levels were already reduced 4 weeks after the drug administration in the group of responders, but not in the group of non-responders (Fig. 3). This observation supports the view that tocilizumab is able to reduce the prothrombotic state of RA only if a reduction of inflammation and of disease activity is obtained. The procoagulant state of RA patients revealed by the high plasma levels of $\mathrm{F} 1+2$ is considered the effect of proinflammatory cytokines, which can be counteracted by IL- 6 blockade and was also observed after TNF inhibition $[5,8]$. Accordingly, we observed a positive correlation between markers of prothrombotic state and inflammation. Actually, the levels of CRP, a reliable marker of inflammation, were not decreased in a relatively high number of patients (6 out of 15) 4 weeks after tocilizumab use. However, these data are in agreement with those reported by other authors, such as Wang et al. [32] and Gabay et al. [33], the latter reporting a response rate to tocilizumab of $40 \%$ (DAS28 remission).

The main limitation of our study is the small number of patients, which was due to the difficulty in finding patients starting a new treatment with tocilizumab after an appropriate washout from other biological therapies. However, this sample size allowed us to obtain a statistical power of $80 \%$, with an alpha error of $5 \%$, and to gather a sample of patients with active disease, naïve for tocilizumab treatment. Indeed, Mokuda et al. stated that RA patients treated with tocilizumab had lower levels of
FXIII than normal controls, but they were in maintenance treatment and had low DAS28 values [10]. In contrast, our patients before the administration of tocilizumab were experiencing an acute phase of the disease with high DAS28 values and CRP plasma levels.

Another limitation was the short time of follow-up (4 weeks); therefore, we cannot exclude that a longer period of treatment may lead to a different response rate and prothrombotic state.

A peculiarity of our study is that we were able to compare a group of patients responding to the drug with a group of patients not responding to the drug. The observation that only responders to the drug had a reduction of FXIII and $F 1+2$ suggests that the reduction of the prothrombotic state exerted by tocilizumab is linked to the regulation of inflammation and disease activity and not to a direct effect of the drug.

\section{CONCLUSIONS}

In RA patients, we observed a decrease of FXIII and $\mathrm{F} 1+2$ levels after tocilizumab treatment only in those patients who responded to the drug. This may indicate that the effect of tocilizumab on the prothrombotic state is due to the control of inflammation and disease activity and not to a direct action of the drug. The effect of tocilizumab on FXIII may be a further mechanism contributing to the reduction of the cardiovascular risk that is one of the major causes of morbidity and mortality in RA patients.

\section{ACKNOWLEDGEMENTS}

We thank the participants of the study.

Funding. Roberta Gualtierotti was supported by a grant provided by Fondazione Cariplo (no. 2017-1938) in collaboration with Fondazione Regionale per la Ricerca Biomedica. No Rapid Service Fee was received by the journal for the publication of this article. 
Authorship. All named authors meet the International Committee of Medical Journal Editors (ICMJE) criteria for authorship for this article, take responsibility for the integrity of the work as a whole, and have given their approval for this version to be published.

Disclosures. Roberta Gualtierotti, Francesca Ingegnoli, Massimo Boscolo, Samantha Griffini, Elena Grovetti and Massimo Cugno have nothing to disclose.

\section{Compliance with Ethics Guideli-} nes. Authors have received Ethics committee approval on 20th July 2017 (Fondazione IRCCS $\mathrm{Ca}^{\prime}$ Granda Ospedale Maggiore Policlinico di Milano, nr. 484_2017). The study conformed with the Helsinki Declaration of 1964, as revised in 2013, concerning human and animal rights, and Springer's policy concerning informed consent has been followed.

Open Access. This article is distributed under the terms of the Creative Commons Attribution-NonCommercial 4.0 International License (http://creativecommons.org/licenses/ by-nc/4.0/), which permits any noncommercial use, distribution, and reproduction in any medium, provided you give appropriate credit to the original author(s) and the source, provide a link to the Creative Commons license, and indicate if changes were made.

\section{REFERENCES}

1. Smolen JS, Aletaha D, McInnes IB. Rheumatoid arthritis. Lancet. 2016;388:2023-38.

2. Nurmohamed MT, Heslinga M, Kitas GD. Cardiovascular comorbidity in rheumatic diseases. Nat Rev Rheumatol. 2015;11:693-704.

3. Cugno $\mathrm{M}$, Ingegnoli $\mathrm{F}$, Gualtierotti $\mathrm{R}$, Fantini $\mathrm{F}$. Potential effect of anti-tumour necrosis factor-alpha treatment on reducing the cardiovascular risk related to rheumatoid arthritis. Curr Vasc Pharmacol. 2010;8:285-92.

4. Roubille C, Richer V, Starnino T, McCourt C, McFarlane A, Fleming $P$, et al. The effects of tumour necrosis factor inhibitors, methotrexate, non- steroidal anti-inflammatory drugs and corticosteroids on cardiovascular events in rheumatoid arthritis, psoriasis and psoriatic arthritis: a systematic review and meta-analysis. Ann Rheum Dis. 2015;74:480-9.

5. Ingegnoli F, Fantini F, Favalli EG, Soldi A, Griffini S, Galbiati $\mathrm{V}$, et al. Inflammatory and prothrombotic biomarkers in patients with rheumatoid arthritis: effects of tumor necrosis factor-alpha blockade. J Autoimmun. 2008;31:175-9.

6. Ingegnoli F, Fantini F, Griffini S, Soldi A, Meroni PL, Cugno M. Anti-tumor necrosis factor alpha therapy normalizes fibrinolysis impairment in patients with active rheumatoid arthritis. Clin Exp Rheumatol. 2010;28:254-7.

7. Singh S, Fumery M, Singh AG, Singh N, Prokop LJ, Dulai PS, et al. Comparative risk of cardiovascular events with biologic and synthetic disease-modifying anti-rheumatic drugs in patients with rheumatoid arthritis: a systematic review and metaanalysis. Arthritis Care Res (Hoboken). 2019. https://doi.org/10.1002/acr.23875.

8. Gualtierotti R, Ingegnoli F, Griffini S, Grovetti E, Meroni PL, Cugno M. Prothrombotic biomarkers in patients with rheumatoid arthritis: the beneficial effect of IL-6 receptor blockade. Clin Exp Rheumatol. $2016 ; 34: 451-8$.

9. Matsuoka M, Majima T, Onodera T, Ieko M, Souri $\mathrm{M}$, Ichinose A, et al. Hemorrhagic-acquired factor XIII deficiency associated with tocilizumab for treatment of rheumatoid arthritis. Int J Hematol. 2012;96:781-5.

10. Mokuda S, Murata Y, Sawada N, Matoba K, Yamada A, Onishi $M$, et al. Tocilizumab induced acquired factor XIII deficiency in patients with rheumatoid arthritis. PLoS ONE. 2013;8:e69944.

11. Souri M, Mokuda S, Inanami H, Osaki T, Takasugi $\mathrm{K}$, Ichinose A. Non-autoimmune combined factor XIII $A$ and $B$ subunit deficiencies in rheumatoid arthritis patients treated with anti-interleukin-6 receptor monoclonal antibody (tocilizumab). Thromb Res. 2016;140:100-5.

12. Richardson VR, Cordell P, Standeven KF, Carter AM. Substrates of Factor XIII-A: roles in thrombosis and wound healing. Clin Sci (Lond). 2013;124:123-37.

13. Peyvandi F, Menegatti M, Palla R. Rare bleeding disorders: worldwide efforts for classification, diagnosis, and management. Semin Thromb Hemost. 2013;39:579-84. 
14. Byrnes JR, Wolberg AS. Newly-recognized roles of factor XIII in thrombosis. Semin Thromb Hemost. 2016;42:445-54.

15. Raghu H, Cruz C, Rewerts CL, Frederick MD, Thornton S, Mullins ES, et al. Transglutaminase factor XIII promotes arthritis through mechanisms linked to inflammation and bone erosion. Blood. 2015;125:427-37.

16. Sun H, Kaartinen MT. Transglutaminase activity regulates differentiation, migration and fusion of osteoclasts via affecting actin dynamics. J Cell Physiol. 2018;233:7497-513.

17. Aletaha D, Neogi T, Silman AJ, Funovits J, Felson DT, Bingham CO 3rd, et al. 2010 Rheumatoid arthritis classification criteria: an American College of Rheumatology/European League against rheumatism collaborative initiative. Arthritis Rheum. 2010;62:2569-81.

18. Prevoo ML, van't Hof MA, Kuper HH, van Leeuwen MA, van de Putte LB, van Riel PL. Modified disease activity scores that include twenty-eight-joint counts. Development and validation in a prospective longitudinal study of patients with rheumatoid arthritis. Arthritis Rheum. 1995;38:44-8.

19. Fransen J, van Riel PL. The disease activity score and the EULAR response criteria. Clin Exp Rheumatol. 2005;23:S93-9.

20. van den Oever IA, Sattar N, Nurmohamed MT. Thromboembolic and cardiovascular risk in rheumatoid arthritis: role of the haemostatic system. Ann Rheum Dis. 2014;73:954-7.

21. Balogh L, Katona E, Mezei ZA, Kallai J, Gindele R, Edes I, et al. Effect of factor XIII levels and polymorphisms on the risk of myocardial infarction in young patients. Mol Cell Biochem. 2018;448:199209.

22. Mezei ZA, Bereczky Z, Katona E, Gindele R, Balogh E, Fiatal S, et al. Factor XIII B subunit polymorphisms and the risk of coronary artery disease. Int J Mol Sci. 2015;16:1143-59.

23. Voko Z, Bereczky Z, Katona E, Adany R, Muszbek L. Factor XIII Val34Leu variant protects against coronary artery disease. A meta-analysis. Thromb Haemost. 2007;97:458-63.
24. Kloczko J, Wojtukiewicz M, Bielawiec M, Zarzycka B, Kinalska I. Plasma factor XIII and some other haemostasis parameters in patients with diabetic angiopathy. Acta Haematol. 1986;76:81-5.

25. Kloczko J, Wojtukiewicz M, Bielawiec M, Zuch A. Alterations of haemostasis parameters with special reference to fibrin stabilization, factor XIII and fibronectin in patients with obliterative atherosclerosis. Thromb Res. 1988;51:575-81.

26. Mansfield MW, Kohler HP, Ariens RA, McCormack LJ, Grant PJ. Circulating levels of coagulation factor XIII in subjects with type 2 diabetes and in their first-degree relatives. Diabetes Care. 2000;23:703-5.

27. Bereczky Z, Balogh E, Katona E, Czuriga I, Edes I, Muszbek L. Elevated factor XIII level and the risk of myocardial infarction in women. Haematologica. 2007;92:287-8.

28. Menegatti M, Peyvandi F. Treatment of rare factor deficiencies other than hemophilia. Blood. 2019;133:415-24.

29. Cugno M, Gualtierotti R, Tedeschi A, Meroni PL. Autoantibodies to coagulation factors: from pathophysiology to diagnosis and therapy. Autoimmun Rev. 2014;13:40-8.

30. Finzel S, Kraus S, Figueiredo CP, Regensburger A, Kocijan R, Rech J, et al. Comparison of the effects of tocilizumab monotherapy and adalimumab in combination with methotrexate on bone erosion repair in rheumatoid arthritis. Ann Rheum Dis. 2019;78:1186-91.

31. Plenz A, Fritz P, Konig G, Laschner W, Saal JG. Immunohistochemical detection of factor XIIIa and factor XIIIs in synovial membranes of patients with rheumatoid arthritis or osteoarthritis. Rheumatol Int. 1996;16:29-36.

32. Wang J, Devenport J, Low JM, Yu D, Hitraya E. Relationship between baseline and early changes in C-reactive protein and interleukin- 6 levels and clinical response to tocilizumab in rheumatoid arthritis. Arthritis Care Res (Hoboken). 2016;68:882-5.

33. Gabay C, Kavanaugh A, Investigators A. Tocilizumab versus adalimumab for rheumatoid arthritisauthors' reply. Lancet. 2013;382:395. 